Alois Woldan

\title{
Lemberg als Antemurale christianitatis zur Zeit des Ersten Weltkriegs
}

Die Vorstellung vom "Bollwerk der Christenheit" ("antemurale christianitatis") ist seit dem Mittelalter verbreitet und wurde auf verschiedene Größen bezogen, auf Staaten, Territorien, aber auch Städte. Besonders häufig findet sich diese Idee in Verbindung mit einer Stadt, die in verschiedenen nationalen Traditionen verschiedene Namen hat: griechisch/lateinisch Leopolis, deutsch Lemberg (von Löwenburg), polnisch Lwów, ukrainisch L'viv, russisch L'vov und jiddisch Lemberik. Alle diese Bezeichnungen meinen den selben geographischen Ort, haben also denselben außersprachlichen Referenten, sie haben aber unterschiedliche Bedeutungen aufgrund unterschiedlicher Konnotationen, die im Lauf einer Jahrhunderte langen Entwicklung mit den einzelsprachlichen Bezeichnungen entstanden sind. Schon von daher ergeben sich auch Probleme bei der Übersetzung der nationalsprachlichen Namensformen: auch wenn von der Etymologie her pol. Lwów und ukr. L'viv adäquate Formen sind, so sind sie dennoch nicht ersetzbar: gerade mit diesen beiden Formen des Stadtnamens sind konkurrierende Vorstellungen von dieser Stadt verbunden, die einander zu einem guten Teil ausschließen. Aber auch das spätmittelalterliche "Löwenburg" meint eine andere Existenzform dieser Stadt, als etwa das österreichische "Lemberg" des 18. Jahrhunderts, wenngleich beide Formen dieselbe Etymologie und Wortbedeutung haben. Es ist also nicht verwunderlich, daß unter den zahlreichen Publikationen, die dieser Stadt gewidmet sind, mehrere auch die Problematik der verschiedenen Namen schon im Titel ansprechen (Fäßler, Held, Sawicki 1995; Voznjak 2003; Balyns'kyj, Matijaš 2008).

Die Stadt mit diesen vielen Namen ist aber nicht nur Gegenstand wissenschaftlicher Untersuchungen, sie ist seit Jahrhunderten auch Objekt der literarischen Beschreibung und kreativen Fiktion. Faßt man alle die Texte zusammen, die auf diese Stadt Bezug nehmen, so läßt sich - in Analogie zum "Petersburger Text der russischen Literatur" - ein Stadttext von Lemberg rekonstruieren ${ }^{1}$, wobei außer Zweifel steht, daß die Rekonstruktion immer auch Konstruktion ist. Gemeinsam ist allen Texten, die diesem Stadttext angehören, daß sie die Stadt selbst zum Thema machen, indem sie auf deren Besonderheiten eingehen. Eine dieser Besonderheiten, die damit auch zur verbindenden Klammer im System des Stadttextes von Lemberg wird, ist die Vorstellung vom "antemurale christianitatis", die in den Jahren vor, während und nach dem Ersten Weltkrieg besonders häufig vorkommt.

1 Ein Versuch einer solchen Rekonstruktion findet sich in Woldan 2008. 
Wenn der Text der Stadt Petersburg aber vor allem in russischer Sprache vorliegt, so ist der Stadttext von Lemberg deutlich mehrsprachig - um 1900 ist er vor allem in deutschen, polnischen und ukrainischen Versionen greifbar, deren innerer Zusammenhang für die komparatistische Analyse eine besonders lohnenswerte Aufgabe darstellt. Die Rekonstruktion des Stadttexts kann also von Bedeutungen ausgehen, welche der Stadt zugeschrieben werden und die den jeweiligen Stadtnamen mit Konnotationen bereichern, bisweilen auch überlagern ${ }^{2}$. Solche Bedeutungen sind in der Regel beständig, was deren Vorkommen über einen längeren Zeitabschnitt betrifft, und auch, was deren Existenz in unterschiedlichen nationalen Narrativen angeht. Vorstellungen wie die von "Leopolis - antemurale christianitatis" bzw. "Leopolis semper fidelis" gehören zu jenen transnationalen Ideen, welche den Text der Stadt über die Jahrhunderte hinweg bestimmen.

Ein eindrucksvolles Beispiel für die Relevanz solcher Vorstellungen stellt ein 1986 in London erschienenes Erinnerungsbuch dar, das schon mit seinem Titel auf die Macht der Imagination verweist: Lwów w naszej pamięci meint eine Stadt, die nur mehr in der Erinnerung ihrer ehemaligen Bewohner (und deren Nachkommen) lebt, die mit der heutigen ukrainischen Stadt L'viv nichts zu tun hat, die aber ein bestimmtes Vermächtnis, den "Geist der Freiheit”, auch an jene Menschen weitergeben kann, die nicht von dort stammen:

Album Lwów w naszej pamięci odtwarza wizję tego wyjątkowego kresowego miasta. Siedem wieków historii jest nim objętych. Przemawiają te wieki dumnie nie tylko do nas, Polaków wyrzuconych z rodzinnych stron. Ale i do obcych, i do tych, którzy znali i poznali to "zawsze wiernie" miasto. Jak długo żyje w naszej pamięci, przetrwa najgorsze nawet chwile, a z tych murów i kamieni, i naszych serc odrodzi sie jego dawny duch wolności (Baraniecki 1986).

Es verwundert nicht, daß im Kontext des "semper fidelis" ("zawsze wiernie") und des Geistes der Freiheit" ("duch wolności") auch die Vorstellung des Antemurale auftaucht für die virtuelle Konstruktion der Stadt, die nur mehr in der Erinnerung einer bestimmten Gemeinschaft lebt, ist auch diese Vorstellung von besonderer Wichtigkeit, die nun nicht mehr an die Situation des Ersten Weltkriegs gebunden ist und damit zum überzeitlichen Charakteristikum der Stadt werden kann: "Lwów, będąc stolicą wschodnich rubieży Rzeczypospolitej, ... ochraniał i odpierał nawały ze Wschodu, a w czasach największych ucisków germanizacyjnych i rusyfikacyjnych był arką, gdzie skryła się i przetrwała polskość" (Baraniecki 1986).

Die Vorstellung vom Bollwerk an der östlichen Grenze, das feindliche Stürme aus dem Osten, von jenseits dieser Grenze, abhält, ist hier mit einer zusätzlichen Idee verbunden, der Arche, die ein bestimmtes nationales Element gegenüber anderen bewahrt und be-

2 Solche Zuschreibungen lassen sich im Sinne von Roland Barthes als Mythen verstehen, als Ergebnisse eines semiotischen Prozesses, bei dem eine vorhandene Bedeutung - der Name der Stadt - zum Zeichen wird, zum Träger einer neuen Bedeutung, die auf der Ebene der Konnotation aufbaut und die ursprüngliche Denotation verdrängt. 
schützt. Dabei werden zwei unterschiedliche räumliche Modelle kontaminiert: im ersten Fall geht es um eine Grenze zwischen West und Ost, die vor dem Osten, der als gefährlich und feindlich angesehen wird, schützt; im zweiten Fall, dem der Arche, geht es um einen isolierten Raum, der ringsum von einer feindlichen Umwelt umgeben ist - Sicherheit und Schutz sind nur in diesem isolierten Raum gegeben, die spätestens seit der Aufklärung bestehende Dichotomie von Ost und West wird nicht angesprochen.

Es liegt auf der Hand, daß diese Modelle aus unterschiedlicher nationaler Perspektive in bezug auf ihre räumlichen Dimensionen unterschiedlich lokalisiert und von verschiedenen Protagonisten besetzt werden können. Nicht nur die Feinde, die von jenseits der Grenze die "christianitas" bedrängen, können verschiedene sein, auch die Protagonisten, die von diesseits der Grenze diese Abwehr leisten, können variieren - so können an die Stelle des unpersönlichen polnischen Lwów die ukrainischen Kosaken treten, die sich ebenso als "antemurale" verstehen. Das wird - lang schon vor 1914 - aus einem Brief des Hetmans Mazepa an den österreichischen Kaiser Joseph I aus dem Jahr 1708 klar. Der ukrainische Hetman begehrt die Würde eines Reichsfürsten mit der Begründung, daß die Kosaken "die ungläubigen Tartern und andere der Christenheit Feinde von denen Christlichen Gräntzen kräfftiglich abgehalten... [hätten] und auch in Zukunft bereit [wären] als eine Vormauer der gantzen Christenheit uns willig bey allen Vorfallenheiten gebrauchen zu lassen" (Mac'kiv 1988: 241)

Die Feinde, die es von der Christenheit abzuhalten gilt, werden explizit genannt Tataren und sonstige Andersgläubige. Die Grenzen und damit auch die Lage der Vormauer sind in diesem Fall nicht geographisch fixiert (im Unterschied zu Lwów und dem östlichen Grenzland der polnischen Adelsrepublik im Beispiel oben), sie könnten bestenfalls aus den Feldzügen Mazepas gegen Türken und Tataren rekonstruiert werden. Aber auch Angaben wie "stolica wschodnich rubieży Rzeczypospolitej" beweisen die Fiktionalität der Geographie des Antemurale: in den Zeiten der Rzeczpospolita, bis zur Ersten Teilung Polens 1772, lag Lwów ganz und gar nicht an der östlichen Grenze der polnischen Adelsrepublik, sondern mitten in diesem Staat, dessen östliche Grenze am Fluß Dnepr verlief.

Auch aus ukrainischer Sicht ist eine genaue Lokalisierung der Grenze zwischen der "christianitas" und einem Bereich jenseits, östlich davon, kaum möglich. "U-kraina" ist schon von der Etymologie her das "Land an der Grenze", das ganze Land ist also Grenze, nicht im Sinne einer exakt gezogenen politischen Grenze (eine solche verlief lange Zeit mitten durch das Land), sondern als ein Grenz- bzw. Übergangsgebiet zwischen West und Ost, zwischen Rom und Byzanz, um einen bekannten Buchtitel zu paraphrasieren (Winter 1942). Somit läßt sich nur schwer sagen, wo das Bollwerk gegen die Feinde der Christenheit zu stehen hätte, im Osten, Süden oder gar im Westen der Ukraine.

Generell ist zu sagen, daß die ukrainischen Belege für das "antemurale" im Vergleich mit den polnischen in der Minderzahl sind, daß die Rede vom Bollwerk ein spezifisch polnischer Diskurs ist, der nur in seltenen Fällen von der ukrainischen Seite angeeignet

3 Diesen Brief hat als erster Theodor Mac'kiv entdeckt und publiziert (Mac'kiv 1988). 
wurde ${ }^{4}$. Der polnische Diskurs des Antemurale (wie auch des "semper fidelis") stützt sich im Wesentlichen auf eine entsprechende Interpretation folgender historischer Fakten:

- 1648 Belagerung der Stadt durch die Truppen Bohdan Chmel'nyckyjs

- 1672 Belagerung durch die Türken

- 1704 Belagerung, Einnahme und Plünderung durch die Schweden unter Karl XII

- 1914 Einnahme durch russische Truppen im Ersten Weltkrieg.

Im 20. Jahrhundert taucht der Topos vom "Bollwerk" schon vor dem Ersten Weltkrieg auf, im Zusammenhang mit einer Institution, bei der man am wenigsten eine militärisch-strategische Funktion vermuten würde, der Universität Lemberg, die im Jahr 1911 ihr 250-jähriges Jubiläum feierte. In diesem Zusammenhang erschien auch eine von Franciszek Jaworski verfaßte Festschrift mit dem Titel Uniwersytet Lwowski, Wspomnienie Jubileuszowe, in der man lesen kann: "[Alma Mater lwowska - A.W.] Jest potężną twierdzą ducha polskiego, czujną strażnicą u wschodnich rubieży Polski, jest jasnem ogniskiem wiedzy, prawdy i kultury..." (Jaworski 1912: 9).

Einmal mehr findet sich hier eine pseudo-militärische Terminologie ("potężna twierdza", "czujną strażnica"), um eine ganz und gar nicht militärische Funktion zu illustrieren die bildungspolitische Funktion der Universität Lemberg, welche aber im Zusammenhang mit deren Lage "an den östlichen Randgebieten Polens" ("u wschodnich rubieży Polski") eine besondere Brisanz gewinnt: nun repräsentiert das Bollwerk auch "Wissen, Wahrheit und Kultur" ("wiedza, prawda, kultura”) als Qualitäten dieses Raums, den es zu verteidigen hat; und es liegt auf der Hand, daß der entgegengesetzte Bereich jenseits der Grenze über diese Qualitäten nicht verfügt. Wenn man bedenkt, daß nur wenige Jahre zuvor der polnisch-ukrainische Konflikt an dieser Universität in der sog. "Blutigen Immatrikulation" von 1907 eskaliert war, die Verhaftungen und Repressionen gegen die ukrainischen Studenten zur Folge hatte, was auch ein weltweites Echo fand, dann zeigt sich, daß der Antemurale-Diskurs nicht nur Gegensätze konstruiert und verfestigt, sondern auch zu deren Verschleierung dient - im Bollwerk selbst, sei es in der Stadt Lemberg oder an deren Universität, darf es im Rahmen dieses Diskurses keine Gegensätze geben; was nicht in dieses Bild paßt, wird mit Schweigen übergangen, von der Forderung nach einer eigenen ukrainischen Universität ist in der erwähnten polnischen Schrift natürlich keine Rede.

Mit dem Ausbruch des Ersten Weltkriegs veränderte sich die Situation radikal, zwei von den Völkern Galiziens - Ukrainer und Polen - kämpften auf beiden Seiten der Front, und beide verbanden große Hoffnungen mit einem Sieg der Mittelmächte bzw. der Niederlage des zaristischen Unterdrückers. Während deutsche Armeen bei Tannenberg in Ostpreußen im August 1914 einen großen Sieg errangen, mußten sich die österreichischen Truppen in Galizien zurückziehen. Schon im September 1914 wurde Lemberg von russi-

4 Einige wenige Beispiele dafür bringt Anna Veronika Wendland 2002. 
schen Truppen besetzt und es brach einmal mehr eine schwere Probe für die Stadt mit dem Anspruch des "semper fidelis" herein.

Unter den vielen literarischen Texten, die diese Zeit thematisieren, zahlreichen Broschüren heute oft vergessener Autoren, die in den verschiedensten Sprachen schrieben, findet sich auch eine Sammlung von Erzählungen mit dem "Bollwerk" im Titel: Galizien. Der Wall im Osten. Kriegserzählungen (1915) von Hermann Blumenthal (1880-1942), einem der vielen jüdischen Autoren aus Ostgalizien, die deutsch geschrieben haben und heute noch auf ihre Wiederentdeckung warten ${ }^{5}$.

Die längste Erzählung aus diesem Band, betitelt mit Die Schlacht bei Lemberg zeigt, wie die österreichischen Behörden die Hauptstadt Galiziens vor den heranrückenden russischen Truppen räumen müssen. Für den Erzähler und seine Protagonisten besteht jedoch die Gewißheit, daß diese Besetzung nur von kurzen Dauer sein wird und letzten Endes die Österreicher siegen werden, denn sie verkörpern eine Macht der Kultur ganz im Gegenteil zu den russischen Truppen, vor allem den Kosaken, die nur Chaos und Vernichtung bringen. Aus der Perspektive des jüdischen Autors ist die österreichische Herrschaft die einzige Garantie gegen die Übergriffe gegen die jüdischen Bevölkerung, welche von den Kosaken im Besonderen, aber auch von den russischen Truppen generell verfolgt wird.

Der jüdische Autor geht auch auf die anderen ethnischen Gruppen in Galizien ein, er konstatiert die Loyalität der Polen, obwohl seine Sympathien eigentlich den Ruthenen (Ukrainern) gehören, wobei er aber zu unterscheiden weiß zwischen jungen Intellektuellen, die sich freiwillig zu den ukrainischen Schützeneinheiten melden, und jenen Bauern, die mit den russischen Truppen kollaborieren, weil sie von der russischen Propaganda gekauft worden sind; immer wieder schildert er die Hinrichtung solcher Bauern, die für die russische Armee Spionagedienste geleistet haben, wobei sein pro-österreichischer Standpunkt deutlich wird - diese Menschen erleiden eine gerechte Strafe, auch wenn sie Opfer einer Verführung sind. Bevor aber in Blumenthals Erzählung die Kampfhandlungen wirklich begonnen haben, bei der Schilderung der galizischen Landschaft um Lemberg, greift der Erzähler auf die Idee des Antemurale zurück, wenn er schreibt:

... mußte ich daran denken, wie oft auf diesem Boden die Kämpfe gegen die Heiden, die gen Westen ziehen wollten, ausgefochten worden waren. Seit jeher war Galizien der Damm, der Europa vor dem Überfluten der heidnischen asiatischen Scharen bewahrt hatte. Nun sollte hier dem Vordringen der Kosakenhorden Einhalt geboten werden (Blumenthal 1915: 19).

Ganz Galizien ist aus dieser Sicht das Antemurale, das nun expressis verbis zwischen Europa und Asien lokalisiert wird; die russischen Truppen werden eingereiht in die Typologie der Heiden aus Asien. Natürlich weiß Blumenthal, daß die galizische Ostgrenze

5 Blumenthal wurde in Bolechów geboren, besuchte das Gymnasium in Lemberg und kam schon vor Kriegsausbruch nach Wien; er wurde Opfer des Holocaust (Blumesberger et al. 2002: 1096). 
am Fluß Zbrutsch mehr als $1000 \mathrm{~km}$ von der geographischen Grenze zwischen Europa und Asien im Ural entfernt ist, natürlich weiß er, daß die Kosaken orthodox und damit christlich sind, im Antmurale-Diskurs jedoch werden Grenzen konstruiert und Feindbilder geschaffen, indem typologische Ähnlichkeiten hergestellt werden - das Verhalten der Kosaken gegenüber der jüdischen Bevölkerung läßt sich bestens mit Stereotypen aus einer Jahrhunderte alten heidnisch-christlichen Konfliktgeschichte beschreiben.

Noch ausgeprägter ist dieser Tenor in den publizistischen Propagandaschriften aus den ersten Kriegsjahren, wie z.B. der Broschüre Der Weltkrieg und das Ukrainische Problem, deren Verfasser, Ottocar Stauf v. d. March, diese Schrift unter dem vielsagenden Pseudonym "Verax" vor allem zur Aufklärung für die befreundeten deutschen Verbündeten verfaßte. Den Ersten Weltkrieg charakterisiert der Verfasser wie folgt:

Dieser Krieg ist ein Krieg der Kultur und Gesittung gegen Barbarei, Heuchelei und Niedertracht... Asiatische Barbarei mit echt mongolischem Einschlag, moskowitische Finsternis und Knechtschaft, durchsetzt von byzantinischer Heuchelei und Heimtücke, droht über das zivilisierte Europa wie eine Sündflut hereinzubrechen und jahrhundertelange Kulturarbeit zu vernichten (Verax 1915: 3).

Mit aller Deutlichkeit wird jetzt das, was vom galizischen Antemurale aufgehalten werden muß, charakterisiert, wobei der spezifische Diskurs einmal mehr die absonderlichsten Konstruktionen und Kontaminationen ermöglicht: der russische Gegner ist sowohl mongolisch und damit asiatisch (eine Anspielung auf die Mongolenherrschaft in Rußland im Spätmittelalter), er ist aber auch byzantinisch, was die Übernahme der christlichen Tradition betrifft, in der er steht. Aus beiden Wurzeln aber stammen negative stereotype Zuschreibungen: mit Asien die Barbarei, mit Byzanz die Heimtücke. Der AntemuraleDiskurs partizipiert in diesem Fall an anderen europäischen Identitätsdiskursen, die ihr Europa aus der Abgrenzung von diversen nicht- oder halbeuropäischen Bereichen definieren und damit als einen Raum der Zivilisation und Kultur - im Fall des zitierten Beispiels auf die Länder der Mittelmächte eingeschränkt - konstituieren. Galizien ist einmal mehr der Ort, wo ein solcher "asiatischer" Vorstoß aufgehalten werden muß.

Während des Ersten Weltkriegs entstanden diverse Schriften, die speziell über Galizien, das nun als Kriegsschauplatz von allgemeinem Interesse war, berichten sollten, wie etwa A. v. Guttrys Buch Galizien. Land und Leute (1916), das allerdings deutlich einen polnischen nationalen Standpunkt widerspiegelt. Für Guttry, der für den deutschen Leser auch über die polnische Literatur geschrieben hatte, ist Galizien ein "Tummelplatz des polnischen und ruthenischen Wettstreits" (Guttry 1916:3) - nicht mehr Österreich und Rußland, Kultur und Barbarei, stehen hier einander gegenüber, sondern Polen und Ukrainer, wobei die Konnotationen aber, die mit den beiden Seiten verbunden sind, dieselben sind: Polen verkörpert die Zivilisation des Westens und hat die "Aufgabe der Kulturvermittlung an alle zu Polen gehörenden nicht polnischen Länder und an den Osten" (Guttry 1916: 4). Lemberg nimmt in einer solchen Konzeption eine Schlüsselstellung ein: 
An der geschichtlichen und kulturellen Mission Polens nahm also Lemberg, das "ornamentum Regni”, in guten und bösen Tagen lebhaften Anteil. Als Hauptschanze der Grenzmarken, als zäher, fester Schutzwall gegen die zahlreichen Feinde der polnischen Republik, stellte es stets kühn und mutig seinen Mann und gab den anstürmenden Heeren blutigen Empfang (Guttry 1916: 236).

Die Vorstellung des Antemurale ist auch für diese Argumentation prägend, wobei allerdings die "christianitas", welche vom Bollwerk geschützt werden soll, eindeutig auf die alte Adelsrepublik bezogen wird.

Mit der Rückeroberung Lembergs im Juni 1915 nach erbitterten Kämpfen seit Ostern desselben Jahres ist Lemberg nun wieder dort, wo es im Sinn des Antemurale-Diskurses immer war - beim Westen, bei Europa, beim Abendland. Es verwundert nicht, daß in dieser Situation die Rede vom "Bollwerk" neue Aktualität erlangt, wie aus einem Reiseführer über diese Stadt, die man nun offenbar wieder gefahrlos bereisen und besichtigen kann, hervorgeht. Lemberg - Handbuch für Kunstliebhaber und Reisende, ist ein Buch betitelt, das 1916 in deutscher Sprache veröffentlicht wurde, aber einen eindeutig polnischen Akzent aufweist; auch sein Verfasser, Josef Piotrowski, scheint Pole zu sein. Schon im Vorwort von Adolf Szyszko-Bohusz wird Lemberg eng mit dem polnischen Staat verbunden, wozu auch die Idee des Bollwerks dient:

War das Reich [Polen - A.W.] eine Vormauer abendländischer Kultur und Gesinnung, so war Lemberg deren weit vorgeschobene Bastei, die den ersten Anprall der wilden feindlichen Scharen aufzunehmen und zu bestehen hatte. Eine starke Wehr war es gegen die tosende östliche Brandung, aber eine Brücke zugleich zwischen Europa und dem Osten (Szyszko-Bohusz 1916: 1).

Zum ersten Mal erscheint hier das Antemurale nicht nur als Bollwerk (belegt durch Begriffe aus der Sprache des Militärs: "Bastei”, "Wehr”), sondern auch Brücke - neben die Abwehr tritt also auch eine Funktion der Vermittlung, die wir schon einmal im Zusammenhang mit der Rolle der Lemberger Universität gesehen hatten. Die russische Besetzung in den Jahren 1914/1915 wird in der üblichen Terminologie und sehr expressiv dargestellt:

Nach über hundert Jahren überschwemmt wieder die grausam und wütend tosende Flut des Orients die Stadt und das Land. Unter anderen Umständen und Verhältnissen des gewaltsamen Weltsturmes branden nun dieselben verwüstenden Wogen der asiatischen Barbarei und Wildheit, deren erstem Anprall Lemberg und das ganze Polenreich stets ausgesetzt waren, an der offenen und friedlichen Stadt (Piotrowski 1916: 36).

Im Frühjahr 1916, als dieser Reiseführer erschien, war der erste Ansturm der "asiatischen Barbarei” gut überstanden, die Stadt hatte ihre Rolle als Antemurale einmal mehr unter Beweis gestellt, der Diskurs war um ein neues Argument bereichert worden. Jetzt konnte man wieder nach Lemberg reisen, jetzt waren die alten Verhältnisse wiederhergestellt - wenn auch nur für drei Jahre, bis zum Herbst 1918 - doch davon ahnte man 1916 noch nichts. 
Ein Jahr zuvor, als Lemberg noch in russischer Hand war und zum ersten Mal in seiner Geschichte "L'vov" hieß, erschien in Petrograd ein Reiseführer unter dem Titel Staryj Lvov. Auch dieser von Vasilij A. Vereščagin verfaßte Führer konzentriert sich primär auf die kunsthistorische Beschreibung, die häufig von polnischen Quellen übernommen wird - ganz anders aber ist der Leitgedanke des Buchs: "staryj" bedeutet für den Verfasser ganz einfach "russisch", wobei das ukrainische Adjektiv "rus'kyj” einfach mit dem russischen Adjektiv "russkij" gleichgesetzt wird - damit ist alles Ruthenische auch russisch, und folgerichtig kann nur von einer "Rückkehr" des immer schon "russischen" Lemberg" in

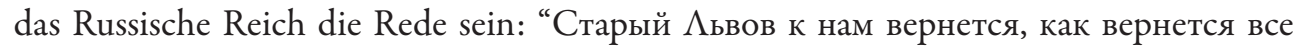
оА нас отторгнутое» (Vereščagin 1915). In dieser Vorstellung scheint die Stadt der am weitesten nach Westen vogeschobene Punkt des russischen Ostens zu sein, der nun wieder mit seinem Mutterland verbunden ist, ganz im Gegenteil zur Vorstellung vom äußersten Bollwerk des (polnischen) Westens.

Den Anspruch auf die polono-zentristische Deutung des Antemurale bestritt die ukrainische Publizistik schon im Ersten Weltkrieg, wobei man sich jedoch auch des Antemurale-Diskurses und ähnlicher räumlicher Modelle bediente. So etwa in zwei Aufsätzen, die Eugen Lewicky, Mitglied des österreichischen Reichsrats und Vizepräsident des Ukrainischen Parlamentarisches Klubs in diesem Parlament, ein typischer "österreichischer Ukrainer" also, 1916 in deutscher Sprache in Berlin unter dem Titel Osteuropäische Probleme und der Krieg publizierte. Auch diese Schriften waren für ein deutsches Publikum gedacht, das von den Anliegen der österreichischen Ukrainer, die nun auch Verbündete auf Seiten der Mittelmächte waren, nur wenig Ahnung hatte. Im zweiten der beiden Aufsätze, Großpolnische Träume und die Wahrheit über die polnische Frage, entwirft der Verfasser ein Bild des zukünftigen Polens - nach einem Sieg der Mittelmächte - , das deutlich an die Vorstellung vom Antemurale anknüpft: "Mit seiner geographischen Lage zwischen drei Großmächten .... kann Polen ... vom internationalen Standpunkte aus nur als ein zukünftiges Grenzland, eine neutrale Scheidemauer in Betracht kommen" (Lewicky 1916: 24). Zugleich aber unterscheidet Lewicky deutlich zwischen den Begriffen "Scheidemauer" und "Vormauer": das, was in der politischen Praxis als eine Art Demarkationslinie funktioniert, hat nichts mit den kulturhistorischen Impliktionen des "antmurale christianitatis" zu tun, sodaß "die Rede von einer "Vormauer der westeuropäischen Kultur", wie wir sie so oft in den Artikeln und Broschüren polnischer Publizisten zu lesen bekommen, nichts mehr als eine Täuschung ist" (Lewicky 1916: 25-26).

Für Polen bleibt aus dieser ukrainischen Sicht nur die Rolle eines neutralen Zwischenfeldes zwischen West und Ost, zwischen Deutschland und Rußland, nicht aber die eines integralen Bestandteils des Westens als dessen äußerstes "Bollwerk". Damit fällt auch die kulturelle Mission Polens für seine östlichen Nachbarn weg: Polen sei nach Meinung des Verfassers kulturell überhaupt nicht so gut entwickelt, daß es eine solche Mission leisten könnte, die Nachbarn wiederum bedienten sich an den Kulturgütern Westeuropas selbst, bräuchten dazu keinen Vermittler. 
Unmittelbar nach Kriegsende begünstigt eine weitere militärische Auseinandersetzung den Antemurale-Diskurs - die Kämpfe um Lemberg im November 1918. Nachdem am 31. Oktober die ukrainischen Schützeneinheiten (Sičovi Stril'cy) die Stadt besetzt hatten, wurde am 1. November dort vom Ukrainischen Nationalrat die Westukrainische Nationale Republik (ZUNR) proklamiert, mit der Hauptstadt L'viv, und damit ein zweiter ukrainischer Staat neben der UNR mit der Hauptstadt Kiev geschaffen. Die überwiegend polnische Bevölkerung der Stadt wurde davon überrascht: es gab nur wenig reguläre Soldaten, um Widerstand zu leisten, der aber bald von Freiwilligen, vor allem Jugendlichen, getragen wurde. Ein Kleinkrieg um einzelne Straßen, Gebäude und Stadtteile entbrannte, den letzten Endes die polnische Seite für sich entscheiden konnte. Nach der Entscheidungsschlacht am 21. November 1918, bei der auch ein polnisches Ersatzkorps aus Przemyśl beteiligt war, zogen sich die ukrainischen Truppen aus der Stadt zurück, um Stanislaviv (heute: Ivano-Frankivs'k) zur neuen Hauptstadt der ZUNR zu machen.

Für Polen war dieser Sieg als ein erster Schritt zur Festigung der neuen Ostgrenze von großer Bedeutung. Unmittelbar nach den Kämpfen vom November 1918 setzte die Mythenbildung ein, die sich auf die Heldentat der "Orłęta Lwowscy", der "Lemberger Jungen Adler", konzentrierte, auf die Jugendlichen, die bei der Verteidigung "ihrer Stadt" gefallen waren, die ihr Leben für ein polnisches Lwów geopfert hatten. Werke über diese jungen Helden erschienen in Publizistik, Lyrik, Drama, Prosa, aber auch in der Bildenden Kunst (Uliasz 2001). Bald entstand auch die Idee eines eigenen Heldenfriedhofs, mit dessen Bau man 1922 begann, und der sich über gut zehn Jahre hinzog. An diesem "campo santo" entstand ein Ort des Gedenkens an Patriotismus und Opferbereitschaft, ein Ort des Kultes, an dem bis 1939 regelmäßig Besichtigungen und Feiern abgehalten wurden (Nicieja 1998: 363-414).

In diesem Kontext wurde gern auf den Diskurs des Antemurale zurückgegriffen. Das soll an einem Beispiel aus der Belletristik gezeigt werden, an Kornel Makuszyńskis Buch Uśmiech Lwowa (1934), einer sentimentalen Geschichte, in der ein vereinsamter alter Herr einen jungen Waisen aus Warschau nach Lwów holt, um ihm alle Schönheiten der Stadt zu zeigen. Diese Führung durch das polnische Lwów kulminiert in jenem Teil des LyczakówFriedhofs, wo die "Orlęta Lwowscy" bestattet sind. Am Grab des eigenen Sohnes, der als Halbwüchsiger im November 1918 gefallen ist, adoptiert der alte Herr seinen Gast: er hat in ihm einen Gleichgesinnten gefunden, der so wie sein Sohn für Lwów einstehen wird. Typisch für das polnische Pathos ist die Rede des alten Herren am Grab:

Tu jest święte miejsce Lwowa... Tu leżą jego dzieci... To jest cmentarz orląt...Naszych dzieci... (Makuszyński 1989: 63)

Był jednak czas, kiedy ci, co leżą w niepoliczonych lwowskich grobach, musieli uczynić rzecz wielką i sprawiedliwą. ... Podpisali swoją krwią manifest o nienaruszalności granic, a granice wytyczyli kopcami swoich grobów. Walczyli na śmierć wbrew nadziei, aby zaświadczyć, żeby idea jest nieśmiertelna i że wobec niej niczym jest ofiara życia. A ta idea, chłopcze, była całość i wielkość Ojczyzny (Makuszyński 1989: 64). 
Wenn auch hier nicht direkt vom "Bollwerk" die Rede ist, so finden sich doch Ähnlichkeiten, die auf denselben Diskurs schließen lassen: die Unverletzlichkeit der polnischen Grenzen, die Idee von der Ganzheit und Größe des Vaterlandes, Polens, das nun anstelle der westlichen Kultur, welche um jeden Preis verteidigt werden muß, steht. Ein zentrales Moment aber im Antemurale-Diskurs fehlt - der Gegner, der vom Bollwerk abgehalten werden soll. Es ist verblüffend, daß bei diesen und ähnlichen Darstellungen der Kämpfe von 1918 kein Wort darüber gesagt wird, gegen wen diese Kinder gekämpft haben, wer die polnischen Grenzen und die Ganzheit des Vaterlands in Frage stellt, gegen wen diese Stadt verteidigt werden muß. Mit Recht spricht George Grabowicz in diesem Fall von der "Dematerialisierung des Anderen” (Hrabovyč 2002: 13), des Ukrainischen, als einer Strategie, welche nur die polnische Sicht der Dinge gelten läßt.

Eine vergleichbare Sicht findet sich auch in anderen Textgattungen aus dieser Zeit, z.B. in Stanisław Wasylewskis Buch Lwów, einer Art Reiseführer, der in einer Serie mit dem bezeichnenden Titel "Cuda Polski” erschien: Auch Wasylewski, der in der Nachkriegszeit eine ganze Reihe von Büchern über Lwów verfaßt hatte, geht auf die Verteidigung der Stadt 1918 ein, die er als einen "Kinderkreuzzug" bezeichnet ("Krucjata Dziecięca" - Wasylewski 1931: 157), ohne daß aber der Gegner in diesem Kampf um das "Heilige Land" genannt würde. Im ganzen Buch werden die Ukrainer so gut wie nicht erwähnt, im Unterschied $\mathrm{zu}$ anderen nicht polnischen Gruppen der Stadtbevölkerung wie Armeniern oder Juden. Lwów als Symbol des wieder erstandenen polnischen Staates braucht seinen multinationalen Charakter bestenfalls als historisches Versatzstück. Die Narration vom polnischen Patriotismus läßt eine ebensolche vom ukrainischen Patriotismus nicht bestehen.

Das Bollwerk ist da, aber der Feind aus dem Osten ist verschwunden. Das große Feindbild aus dem Ersten Weltkrieg, das Zarenreich, besteht nicht mehr - der faktische Gegner, die Ukrainer, aber eignen sich offenbar nicht für diese Rolle der barbarischen Macht aus dem Osten. Sie sind zum einen zu wenig markant, was den Vergleich mit den riesigen Armeen des Zarenreichs oder auch des Osmanischen Imperiums in früheren Jahrhunderten angeht, sie sind aber - und das scheint noch wichtiger zu sein, keine Größe, die von Osten gegen das Bollwerk andrängt, sie sind ja in der Stadt selbst ansässig bzw. kommen aus den umliegenden ländlichen Gegenden, aus jenem Galizien, das auch als Ganzes Bollwerk ist.

Eine gegenteilige Tendenz finden wir in Texten wie Aleksander Medyńskis Reiseführer Lwów. Ilustrowany przewodnik dla zwiedzajacych miasto (1937), die den erfolgreichen Abwehrkampf gegen die Ukrainer von 1918 in eine Reihe mit der Abwehr der Türken im 17. Jahrhundert und der Behauptung gegen die russischen Armeen am Beginn des 20. Jahrhunderts stellt; jetzt erlebt der Diskurs vom Antemurale eine Neuauflage in seiner bekannten Form:

Promiennym ogniskiem polskiej kultury był stary, dostojny gród lwowski, jej szańcem niewzruszonym pozostawał w ciągu wieków. ... Jak fale niezmierzonego oceanu płynęły od wschodnich granic w kierunku tej wspaniałej, kresowej strażnicy skłębione, niszczycielskie watahy i hordy (Medyński 1937: 5). 
... przeciw brutalnej inwazji "ukraińskiej” w imię idei niepodległości i przynależności do Macierzy dźwignął się wspaniały mocarny Czyn Obrońców Lwowa, tych bardzo każdemu polskiemu sercu drogich "Orłów i Orłąt”... (Medyński 1937: 13).

Einmal mehr ist die Rede vom Bollwerk ("szaniec”, "strażnica”), das zugleich auch Zentrum der Kultur ist, einmal mehr ist die Lokalisierung an den östlichen Grenzen gegeben, und einmal mehr sind es zerstörerische Horden, die gegen dieses Bollwerk anrennen, wobei jetzt auch der letzte Feind benannt wird, die Ukrainer, wenngleich diese ja nicht aus dem Osten kommen, sondern schon immer da sind, in der Stadt und in deren nächster Umgebung. Der wirkliche Feind im Osten, die Sowjetunion, die für Polen viel gefährlicher werden sollte, als es die Ukrainer im "eigenen" Land je waren (einen Vorgeschmack davon hatte der polnisch-sowjetische Krieg von 1920 gebracht, als Budennyjs Reiterarmee der Stadt Lwów gefährlich nahe gekommen war), wird in diesem Fall nicht in den AntemuraleDiskurs integriert.

Dagegen finden wir in einer repräsentativen Selbstdarstellung Polens im Polen-Band der Wielka Ilustrowana Encyclopedja Powszechna nicht nur eine Ausweitung des Antemurale-Konzepts auf ganz Polen im Sinne der II. Polnischen Republik, sondern ist mit dem Feind im Osten klar und deutlich das bolschwistische System angesprochen:

... natrafiamy przedewszystkim na drogą sercom naszym nazwę antemurale Christianitatis. Tu zdaje się istotnie leżeć najoczywistsza analogia pomiędzy przeznaczeniem Polski przedrozbiorowej a dzisiejszej ...

My, Polacy, wiemy dobrze, że bolszewizm ze swem materjalistycznem wyznaniem wiary i klasową tyranją polityczną jest tylko najjaskrawszym wyrazem niebezpieczeństwa dla cywilizacji europejskiej (Dyboski 1931: 10).

Das dem Antemurale-Diskurs zugrundeliegende räumliche Modell findet sich auch in der deutschsprachigen Galizien-Literatur, beim bekannten österreichischen Autor Joseph Roth, einem "Parade-Galizier", der das österreichische Lemberg aus seiner Jugend gut kannte, und der wenige Jahre nach dem Ersten Weltkrieg das polnische Lwów als Berichterstatter für deutsche Zeitungen besuchte. In seinem bekannten Essay Stadt der verwischten Grenzen (1924) schreibt Roth:

Es ist die Stadt der verwischten Grenzen. Der östliche Ausläufer der alten kaiserlichen und königlichen Welt. Hinter Lemberg beginnt Rußland, eine andere Welt. Das weit westlichere Krakau ist weniger österreichisch. Es bleibt immer ein nationales Museum. Zwischen Wien und Lemberg ist heute noch, wie immer, der Radioaustausch der Kultur. ... Der Umsturz hat nämlich alle galizischen Städte um einige Meilen nach Osten gerückt. Vielleicht zum Segen des Ostens (Roth 1976: 840).

Einmal mehr liegt dieses Lemberg, das für Joseph Roth auch 1924 nicht Lwów heißt, an der Grenze zwischen zwei Welten, erinnert also an das Bollwerk, aber die Grenzen sind 
verwischt, durchlässig, fördern den Austausch, nicht nur mit Wien, sondern auch mit den östlichen Nachbarn, wie aus der hier zugrundeliegenden geographischen Modellierung hervorgeht: nach dem Ende der alten Welt, mit dem Entstehen der neuen Nationalstaaten Mitteleuropas, sind nämlich die Städte Galiziens "nach Osten gerückt": nicht, um das, was von dort kommt, abzuhalten, sondern, wie der Autor sagt, "zum Segen des Ostens" - wenn hier vielleicht auch noch ein Rest jener abendländischen Mission, die aus polnischer Sicht so typisch für das Antemurale war, mitschwingt, so steht doch einer solchen Auffassung eine neue Sicht gegenüber: alte urbanen Zentren wie Lemberg sind nicht an Nationalstaaten gebunden, sie können aus einem Staat in einen anderen wandern, ohne ihre elementare, kulturelle Funktion einzubüßen.

Noch einmal zurück zu den Kämpfen vom November 1918. Es liegt nahe, daß die ukrainische Publizistik die polnischen Ansprüche auf Lwów, die nach dem militärischen Erfolg von 1918 besonders massiv waren, in der europäischen Öffentlichkeit zu widerlegen trachtete. Die Zahl der ukrainischen Publikationen ist einmal mehr geringer als die der polnischen (der Sieger schreibt die Geschichte, nicht der Verlierer), sie darf aber deswegen nicht übersehen werden, noch dazu, wo sie auch neue Momente in den AntemuraleDiskurs einbringt. 1919, wenige Monate nach der Niederlage der Ukrainer im Kampf um Lemberg, gibt Stefan Smal'-Stoc'kyj, bekannter Sprachwissenschaftler an der Universität Czernowitz, im Prager Exil eine Broschüre in tschechischer Sprache heraus, die also für ein nicht-ukrainisches Publikum, für die Öffentlichkeit in der Masaryk'schen Tschechoslowakei bestimmt war: Lvov, srdce západni Ukrajiny. Beim Versucht, die polnischen Ansprüche auf die Stadt, militärisch durchgesetzt, aber noch nicht politisch sanktioniert, zu widerlegen, greift der Verfasser einmal mehr auf geographische Modelle zurück - Lemberg liegt für ihn mitten im ukrainischen Siedlungsgebiet, eine Tatsache, die sämtliche polnischen Ansprüche zunichte mache: "Dostačcí však pohlédnouti na jakoukoliv, třebas i polskou etnografickou mapu, aby se kde kdo přesvědčil, že Lvov leží ve středu nepopíratelně ukrajinského území. Proto nemohou Poláci, alespoň spravedlivě, dovolávati se svého práva na Lvov..." (Smal-Stoćkyj 1919: 3-4).

Dieses Argument, im 20. Jahrhundert bei weitem nicht nur von den Ukrainern gebraucht, entspricht aufgrund seiner räumlichen Modelliertheit auch der polnischen Vorstellung von Lwów als einem vorgeschobenen, vom Mutterland abgekapselten Posten nur, daß die nationale Besetzung des "Herzens" eine gegensätzliche ist, das polnische Herz muß sich gegen seine ukrainische Umgebung behaupten, das ukrainische ist mit seiner Umgebung auf das engste verbunden, wie auch aus der im Schlußsatz wieder aufgegriffenen Titelmetapher hervorgeht: "Věru, Lvov jest srdce ukrajinského území, a srdce si nedají Ukrajinci ze svého národního těla jakživ a nijak vytrhnouti” (Smal-Stoćkyj 1919: 14).

Die Herz-Metapher taucht übrigens Jahrzehnte später auf der Gegenseite auf: 1993 gibt Danuta Łomaczewska zum 75-jährigen Gedenken der Ereignisse von 1918 eine umfangreiche Anthologie von Lyrik über Lwów heraus, betitelt mit: Serce wydarte z polskiej piersi. Lwów w poezji (Łomaczewska 1993). Nun ist - aus polnischer Sicht - das wahr geworden und im Titel ausgesprochen, was für Smal'-Stock'yj undenkbar war: das Herz 
wurde aus dem lebendigen Organismus, der jetzt ein polnischer und kein ukrainischer ist, herausgerissen.

In die Reihe der ukrainischen Publikationen, die auf die Ereignisse von 1918 reagieren, gehört auch jenes kleine Buch, das 1927 im schon polnischen Lwów in ukrainischer Sprache erschien und anhand eines historischen Abrisses den ukrainischen Charakter der Stadt unter Beweis stellen wollte: Za ukraijns'kyj L'viv (Holubec' 1927). Wohl aus Gründen der Zensur mußte der Verfasser seine Ausführungen auf die Zeit bis 1772 konzentrieren, er verstand es aber zwischen den Zeilen auf die gegenwärtige Situation anzuspielen. Ausgehend von der These, daß sich die Geschichte wiederholt, schildert Holubec' die Situation der Ukrainer in der Stadt in der frühen Neuzeit wie folgt: "Було в ньому мало Русинів, але богато Pусі” (Holubec' 1927: 6). Dann aber kommt das geographisches Argument von Stadt und Umgebung - hinter der ruthenischen Straße in L'viv steht eine ganze ukrainische Welt: "коли поза вірменською улицею не було вже нікого і нічого, то поза руською межав цілий світ, сонний і несвідомий, але засілюючий ії усім тим, що прокинулося зі сну" (Holubec' 1927: 7). Auch wenn man in der Stadt - und hier ist die Anspielung auf das Lwów der Zwischenkriegszeit nicht zu überhören - nur wenig Ukrainisches spürt, so kann sich dieses nationale Element auf ein weites Hinterland stützen und von dort seine Energien beziehen; weil aber die gesamte Umgebung ukrainisch ist, so muß auch L'viv eine ukrainische Stadt sein:

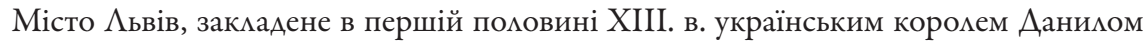
Романовичем на споконвіку українській зелі, було з пріроди річи українським містом і нікому не приходимо на думку боронити його українськости так довго, як Аовго ^асі сусіди не протягнули до нього своїх рук (Holubec' 1927: 11).

Viel Verständnis für die ukrainischen Ansprüche auf Lemberg - auch ohne Kenntnis der zitierten tschechischen und ukrainischen Texte - zeigt der Bericht eines bekannten deutschen Autors, Alfred Döblin, der 1926 seine Reise in Polen veröffentlichte. Im Unterschied zu Joseph Roth, der zwei Jahre vorher Lemberg besucht und beschrieben hatte, bemerkt Döblin sofort die Spannungen zwischen Polen und Ukrainern (Roth, der die Lage viel besser kannte, äußerte sich dazu kaum):

Kasimir der Große hatte die Burg Danillos zerstört und dies neue Lemberg gebaut. Aber die Ukrainer erkennen das polnische Faktum Lemberg nicht an. Das ukrainische Volk lebt zerrissen zwischen Russen und Polen, und es tritt keine Ruhe ein. Ich spreche mit Männern in der Stadt. Ich bekomme den Eindruck eines heimlichen, aber furchtbar intensiven Völkerkampfes (Döblin 1968: 191).

Für Döblin gehen die Kämpfe, die im November 1918 ausgebrochen und deren Spuren er noch an Bauwerken festellen kann, weiter - im Untergrund, aber deswegen nicht weniger gefährlich. Schuld an dem Konflikt trägt aber nicht so sehr die eine oder die andere Seite, sondern das Phänomen des modernen Nationalstaats an sich, der mit seinen 
Grenzziehungen nie den Bedürfnissen multiethnischer Gebiete gerecht werden kann: "Nun haben sie [die Polen - A.W.] einen Staat, und er trägt Gift in ihr Volkstum. Die Grenze schlägt auf sie zurück. Sie haben über sich hinausgegriffen" (Döblin 1968: 200). Hier wird die Kehrseite dessen angesprochen, was Roth mit den "verwischten Grenzen" gemeint hatte: wenn diese Grenzen nicht mehr durchlässig sind, wenn die galizischen Städte nicht mehr über diese Grenzen hinweg wandern können, dann entstehen Konflikte, die bis zur Auseinandersetzung auf Leben und Tod führen können (die Eskalation des polnisch-ukrainischen Konflikts in den 1930er und 1940er Jahren hätte Alfred Döblin Recht gegeben). Und wenn Döblin von "historischen Erinnerungen" spricht, die das Verhältnis zwischen diesen beiden Völkern belasten, so könnten damit auch Konzepte wie das "Antemurale" gemeint sein:

Historische Erinnerungen werden leicht zu Wahnideen. Welche Unmasse gefährlicher Erinnerungen hat jeder europäische Staat. Grausig viel Polen. Aber auch Polens Nachbarn haben Erinnerungen. ... Eine natürliche Symbiose der Völker hier muß sich anbahnen, in besseren loseren Formen als heute (Döblin 1968: 201).

Der Antemurale-Diskurs, eine Konstante im Stadttext von Leopolis, zeigt, wie ein räumliches Modell mit Bedeutungen semiotisch aufgeladen wird. Wir finden wenige elementare Bestandteile dieses Modells - einen dichotomisch in West und Ost geteilten Raum, eine markante Grenze zwischen diesen beiden Teilräumen und ein Bollwerk an der westlichen Seite dieser Grenze, das vor den Einflüssen aus dem Osten schützen soll. Bisweilen variiert dieses räumliche Modell auch dahingehend, daß sich das Bollwerk als eine Art von Insel inmitten eines feindlichen Umlandes befindet, das allerdings im Fall der HerzMetaphorik auch ein homogenes, eigenes Umfeld sein kann.

In jedem Fall aber werden die räumlichen Bestandteile dieses Modells mit konkreten historischen Größen aufgeladen, die variabel sind: der westliche Teil des Raumes kann vom christlichen Abendland, der polnischen Rzeczpospolita, Österreich-Ungarn oder der polnischen Republik der Zwischenkriegszeit besetzt sein, der östliche Teil kann als Basis für den islamischen Orient, das russische Zarenreich oder auch die ukrainische Bevölkerung Galiziens dienen. Und auch das Bollwerk selbst, primär eine Zuschreibung der Stadt Lemberg, kann in seltenen Fällen auf ganz Galizien oder ganz Polen, im Gegenfall auch auf die ganze Ukraine ausgedehnt werden.

So sehr die historisch-politischen Akteure in diesem räumlichen Modell variabel und austauschbar sind, so konstant sind die Konnotationen, die mit den jeweiligen Positionen verbunden sind: der Raum des Westens ist mit Werten der Ordnung und Zivilisation besetzt, der des Ostens mit gegenteiligen Vorstellungen des Chaos und der Barbarei. Das Antemurale selbst verfügt in hohem Maß über die Werte der westlichen Hemisphäre, die es ja zu verteidigen hat. Die auffällige Stereotypie der Wertzuschreibungen, die sich bewußt über historische Fakten hinwegsetzt (wenn etwa die orthodoxen Kosaken mit heidnischen Barbaren gleichgesetzt werden oder die ortsansässigen Ukrainer aus dem Osten einfallen), 
ist typisch für den Diskurscharakter der Rede vom Antemurale. Ähnliches zeigt sich, wenn man das räumliche Modell mit einer realen, politischen Geographie vergleicht: Lemberg lag nie unmittelbar an der östlichen Grenze der jeweiligen Staaten, vor 1772 lag die Stadt mehrere hundert Kilometer von der polnisch-russischen Grenze entfernt, nach 1772 und nach 1918 immerhin noch mehr als hundert Kilometer. Noch viel weniger kann man von der Lage an einer europäisch-asiatischen Grenze sprechen. Hier ist immer geographische Fiktion am Werk, "mental mapping", das eindeutig ideologischen Zwecken dient.

Der Antemurale-Diskurs zeigt auch ein weiteres Moment, das für jeden Diskurs typisch ist - die Verbindung von Rede und Macht. Dieser Diskurs läßt auf den Stärkeren in diesem Sprachspiel zurückschließen, auf die polnische Seite, die ihre Regeln des Diskurses vorgibt, sie zeigt aber auch, wie die schwächere, die ukrainische Seite, versucht, diese Regeln anzueignen und für sich zu nützen (wenn etwa das Modell des Antemurale als einer Insel in feindlicher Umgebung umgedeutet wird zum Zentrum, zum "Herzen" einer homogenen, eigenen Umgebung). Schon die Anzahl der Belege gibt einen ersten Hinweis auf die polnische als die überlegene Seite, ungeachtet dessen, daß so manche polono-zentristische Darstellung nicht in polnischer, sondern in deutscher Sprache verfaßt ist (so wie auch gegenteilige Darstellungen von ukrainischer Seite nicht nur in ukrainischer Sprache vorliegen).

Eine Aufweichung des starren, dichotomischen Modells und eine zumindest ansatzweise Hinterfragung der stereotypen Wertzuschreibungen findet sich bei den beiden erwähnten deutschsprachigen Autoren der Zwischenkriegszeit, Joseph Roth und Alfred Döblin, die beide für die Durchlässigkeit jener fundamentalen Grenze und damit für einen Ausgleich der von dieser Grenze geschiedenen, heterogenen Teilbereiche des Raumes plädieren.

\section{Bibliographie}

Balyns'kyj, Matijaš 2008: I. Balyns'kyj, B. Matijaš (hrsg.), Leopolis multiplex, Kyjiv 2008.

Baraniecki 1986:

J. Baraniecki, Przedmowa, in: Lwów w naszej pamięci. Album naktadem polskiej YMCA w Wielkiej Brytanii i Kota Lwowian w Londynie, London 1986.

Blumenthal 1915:

H. Blumenthal, Galizien. Der Wall im Osten. Kriegserzäblungen, München 1915.

Blumesberger et al. 2002: $\quad$ S. Blumesberger, M. Doppelhofer, G. Mauthe (hrsg.), Handbuch österreichischer Autorinnen und Autoren jüdischer Herkunft 18. bis 20. Jabrhundert, I, München 2002.

Döblin 1968:

A. Döblin, Lemberg, in: Id., Reise in Polen, Freiburg i. B. 1968, S. 181-122.

Dyboski 1931:

R. Dyboski, Przedmowa, in: Wielka Encyklopedja powszechna, XIII (Polska), Kraków 1931, S. 9-14. 
Fäßler, Held, Sawcki 1995: ～P. Fäßler, T. Held, D. Sawitzki (hrsg.), Lemberg - Lwów - Lviv. Eine Stadt im Schnittpunkt europäischer Kulturen, Köln-Weimar-Wien $1995^{2}$.

Guttry 1916:

A. v. Guttry, Galizien. Land und Leute, München 1916.

Holubec' 1927:

Hrabovyč 2002:

M. Holubec', Za ukrajins'kyj L'viv (Epizody borot'by XIII-XVIII v.), L'viv 1927.

H. Hrabovyč, Mitologizaciji L'vova, "Krytyka”, IV, 2002, 7-8, S. 11 17.

Jaworski 1912:

F. Jaworski, Uniwersytet Lwowski, Wspomnienie Jubileuszowe, L'viv 1912.

Lewicky 1916:

E. Lewicky, Osteuropäische Probleme und der Krieg. Zwei Aufsätze, Berlin 1916.

Łomaczewska 1993:

D. B. Łomaczewska, Serce wydarte z polskiej piersi. Lwów w poezji. Warszawa 1993.

Mac'kiv 1988:

Makuszyński 1989:

T. Mac'kiv, Hetman Ivan Mazepa v zachidn'ojevropejs'kich džerelach 1687-1709, München 1988.

Medyński 1937:

K. Makuszyński, Uśmiech Lwowa, Kraków 1989.

A. Medyński, Lwów. Ilustrowany przewodnik dla zwiedzających miasto, Lwów 1937.

Nicieja 1998:

Piotrowski 1916:

S.S. Nicieja, Eyczaków, dzielnica za Styksem, W rocław 1998.

J. Piotrowski, Lemberg und Umgebung (Źótkiew, Podhorce, Brzeżany und and.). Handbuch für Kunstliebhaber und Reisende, Leipzig-Wien 1916.

Roth 1976:

J. Roth, Lemberg, die Stadt, in: J. Roth, Werke, hrsg. von H. Kesten, III, Köln 1976, S. 836-840.

Smal-Stoćkyj 1919:

Š. Smal-Stoćkyj, Lvov, srdce západni Ukrajiny, Kyjiv-Praha 1919.

Szyszko-Bohusz 1916:

A. Szyszko-Bohusz, Vorwort, in: J. Piotrowski, Lemberg und Umgebung (Żótkiew, Podhorce, Brzeżany und and.). Handbuch für Kunstliebhaber und Reisende, Leipzig-Wien 1916, S. 1-2.

Uliasz 2001:

S. Uliasz, $Z$ dziejów legend Ortąt Lwowskich w literaturze polskiej, in: Id., O literaturze kresów i pograniczu kultur. Rozprawy i szkice, Rzeszów 2001, S. 43-64.

Verax 1915:

Verax [O. Stauf v.d. March], Der Weltkrieg und das Ukrainische Problem, Berlin 1915.

Vereščagin 1915:

V.A. Vereščagin, Predislovie, in: Id., Staryj L'vov, Petrograd 1915.

Voznjak 2003:

Wasylewski 1931:

T. Voznjak (hrsg.), Genius Loci: L'viv - Leopolis - Lwów - Lemberg, L'viv 2003 (= "Nezaležnyj kul'turolohyčnyj časopys «Ji»", 2003, 29).

S. Wasylewski, Lwów, Poznań 1931. 
Wendland 2002:

Winter 1942:

Woldan 2008:
A.V. Wendland, Semper fidelis: Lwów jako narodowy mit Polaków $i$ Ukrainców 1867-1939, in: Lwów. Miasto, spoteczeństwo, kultura. Studia z dziejów Lwowa, pod red. K. Karolczaka, IV, Kraków 2002, S. 263-273.

E. Winter, Byzanz und Rom im Kampf um die Ukraine 955-1939, Leipzig 1942.

A. Woldan (hrsg.), Europa Erlesen. Lemberg, Klagenfurt 2008.

\section{Abstract}

\section{Alois Woldan}

The Notion of Antemurale Christianitatis in Connection with the City of Lemberg/Lwów/L 'viv

"Antemurale christianitatis" ("Bastion of Christianity") is a notion frequently associated with the city of L'viv / Lwów/ Lemberg / Leopolis , especially within the context of World War I and the following years. This notion occurs in texts of different genres written in different languages from different national points of view. This concept is thus an essential element of the text of the city of L'viv. The concept of "antemurale" is based on a spatial model consisting of a few elementary components - a dichotomous space divided into two parts, West and East, a strict border between them, and the bastion itself as a kind of fortress situated to the West of that border . In the given texts, all these components are displayed by different historic actors, political powers which are to fulfil either the role of the West or that of the East. When the actors in this concept change, the connotations connected with certain roles do not change - the West is always civilized and cultured, the East always chaotic and barbaric, while the bastion has to defend pure Western values. Here we can find stereotypes which do not correspond to real facts, but show the imaginative force of the concept. Discussing the notion of "antemurale" leads to a discourse that shows the influence of political power executed by linguistic expressions: the concept of "antemurale" is mainly a Polish invention, but it is taken over by the weaker opponent, the Ukrainian side in Galicia, which takes over this model to display it in another, controversial way. There is only a very small number of voices, Austrian and German writers, who try to weaken the rigid border line between the two antagonistic spaces and in this way reveal the notion of Antemurale as an ideological concept.

\section{Keywords}

Antemurale; L'viv; World War I. 